\title{
From DNA to proteins via the ribosome: Structural insights into the workings of the translation machinery
}

\author{
Xabier Agirrezabalal and Joachim Frank ${ }^{2,3}$ \\ 'Structural Biology Unit, CIC-bioGUNE, Bizkaia Technology Park, Derio 48160, Spain \\ ${ }^{2}$ HHMI, Department of Biochemistry and Molecular Biophysics, Columbia University, 650 West 168th Street, P\&S BB 2-221, \\ New York, NY 10032, USA \\ ${ }^{3}$ Department of Biological Sciences, Columbia University, New York, NY 10027, USA \\ *Correspondence to: Tel: ?; Fax: ?; E-mail: ?
}

Date received (in revised form): 13th April, 2010

\begin{abstract}
Understanding protein synthesis in bacteria and humans is important for understanding the origin of many human diseases and devising treatments for them. Over the past decade, the field of structural biology has made significant advances in the visualisation of the molecular machinery involved in protein synthesis. It is now possible to discern, at least in outline, the way that interlocking ribosomal components and factors adapt their conformations throughout this process. The determination of structures in various functional contexts, along with the application of kinetic and fluorescent resonance energy transfer approaches to the problem, has given researchers the frame of reference for what remains as the greatest challenge: the complete dynamic portrait of protein synthesis in the cell.
\end{abstract}

Keywords: ribosome, protein synthesis, structure, dynamics, cryo-EM

\section{Introduction}

The translation of the genetic code is one of the most crucial (and energy-costly) processes of life. This task is performed by ribosomes - large ribonucleoprotein assemblies that read the message encoded in the messenger RNA (mRNA) and synthesise proteins by sequential polymerisation of amino acids carried by transfer RNAs (tRNAs), in the form of aminoacyl-tRNAs (aa-tRNAs). The functional complexity of the protein translation process is reflected by the structural complexity of the ribosome, as it is composed of more than 50 different proteins and several RNA components, even in the simplest organisms. The recent award of the Nobel Prize to three X-ray crystallographers, Venki Ramakrishnan, Tom Steitz and Ada Yonath, amounts to a recognition not only of outstanding individual scientific achievements, but also of the pre-eminent role of this molecular machinery in all forms of life. The architectural sophistication and functional virtuosity of the ribosome are astounding, especially considering that it is one of the most ancient macromolecules, having evidently emerged at the very earliest stages of evolution.

The four major steps during protein synthesis by the ribosome are initiation, elongation, termination and recycling (Figure 1). Briefly, in the first step, ribosomal subunits, mRNA and the initiator tRNA (fMet-tRNA ${ }^{\mathrm{fMet}}$ ) are brought together with the help of initiation factors. Once this initiation complex is formed, the sequential incorporation of aa-tRNAs proceeds based on the cognate match between the codon, a three-base sequence on the mRNA, with the complementary anticodon of the 


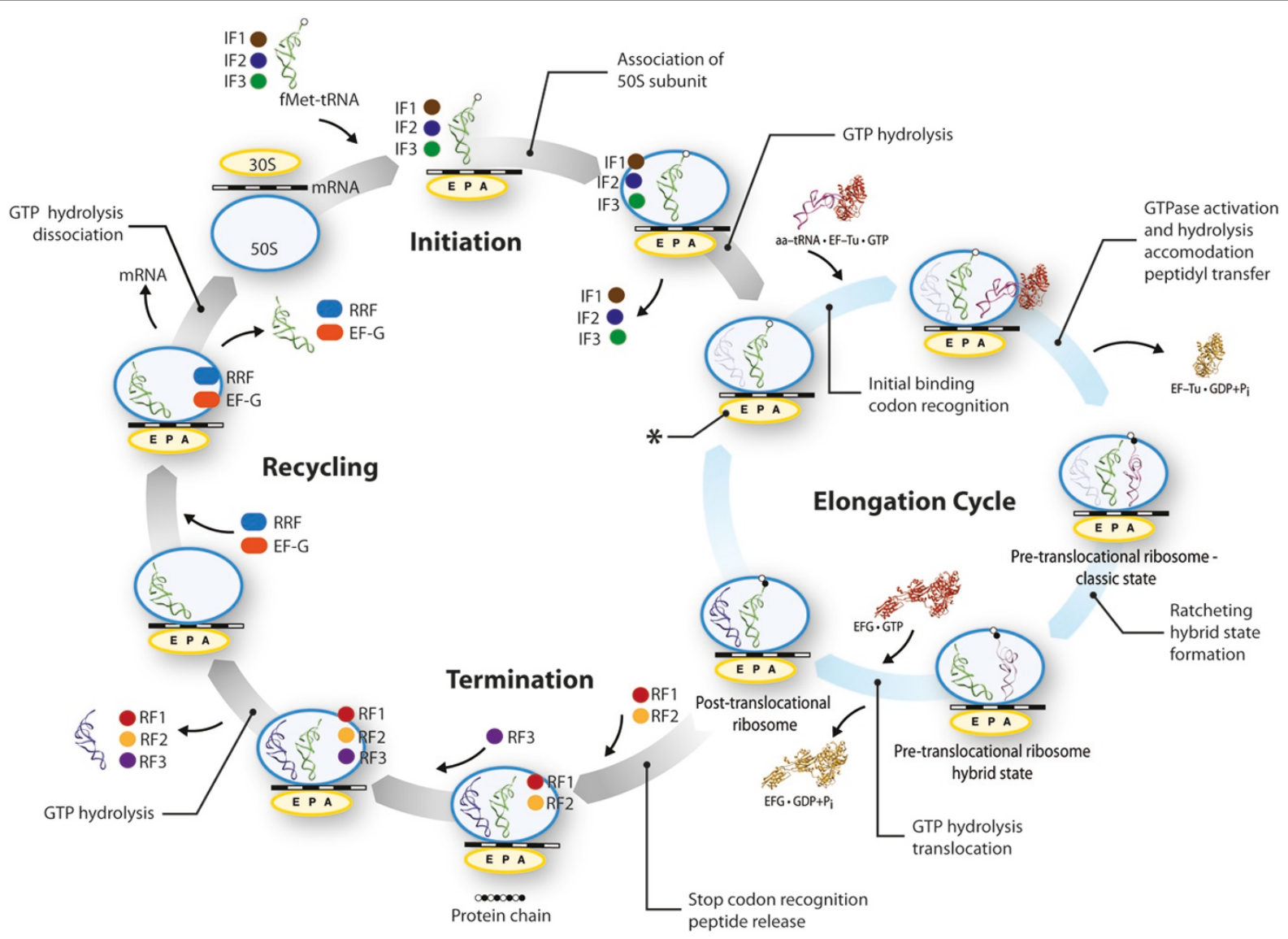

Figure I. Schematic diagram of bacterial protein synthesis. Shown are the mains steps comprising the translation process: initiation, elongation cycle, termination and recycling. Details regarding each step are provided in the main text. For simplicity, some intermediate stages are omitted in this overview. The mRNA is depicted as a strand running horizontally along the small (30S) subunit, with alternating white and black segments, each representing one codon. The tRNAs bind at A, P and E sites. The nascent polypeptide is shown as a string of spheres. The individual structures and cartoons are not drawn to scale.

aa-tRNA, a match that is recognised by the decoding centre located on the small subunit. Upon successful selection, the aa-tRNA is accommodated in the ribosome, leading to the immediate transfer of the peptide bond from the peptidyl tRNA to the incoming aa-tRNA. Next, the tRNAs along with the mRNA, are translocated by the span of one codon, and the decoding site is vacated for the next round of decoding. In each cycle, the assistance of two GTPases is needed, elongation factor (EF)-Tu and EF-G. While aa-tRNA accommodation is guided by EF-Tu (eEF1A in eukaryotes), mRNA-tRNA translocation is promoted by EF-G (eEF2 in eukaryotes). By contrast, peptide transfer is catalysed by the ribosome itself, without the aid of a factor. Once a stop codon of the mRNA reaches the decoding centre, release factors liberate the polypeptide from the ribosome. In the last step, recycling factors (with assistance from EF-G) act to dissociate the ribosome, allowing each component to be re-used.

The structure of the ribosome is defined by the architecture and arrangement of its distinctly sized subunits, large and small, whose association is mediated by several inter-subunit bridges. In eubacteria and archaea, the small and large subunits are referred to by their sedimentation values as $30 \mathrm{~S}$ and 50S, respectively, while their eukaryotic counterparts are designated $40 \mathrm{~S}$ and 60S. Due to the unique topology of the two subunits, their association results in the formation of $\mathrm{a} \sim 100-\AA$ long cavity open on both sides, the inter-subunit space. 
The tRNAs employ this cavity to traverse the entire ribosome as they pass the different primary binding sites (termed A for amino-acyl, P for peptidyl and $\mathrm{E}$ for exit). The cyclic protein elongation process, encompassing decoding, peptide bond transfer and translocation, relies on universally conserved mechanisms. Indeed, the functional centres of the ribosome (ie decoding and peptidyltransferase centres) are located centrally, with highly conserved tertiary structure and disposition.

In contrast to elongation, the processes of initiation, termination and recycling diverge much more among the different kingdoms, and are in general significantly more complex in eukaryotes compared with their bacterial counterparts. This is especially true for initiation. While eubacteria accomplish this task with the aid of three factors (initiation factor [IF]1, IF2 and IF3), initiation in eukaryotes is an intricate, highly regulated process in which more than ten different factors, some of which are multi-subunit complexes, are required. ${ }^{1}$ This high degree of complexity is also reflected in the biogenesis of the subunits, a process that requires the assistance of hundreds of accessory proteins and small nucleolar ribosomal RNAs (rRNA) in eukaryotic systems. In addition, the process is highly compartmentalised: the first steps occur in the nucleolus, and the pre-ribosomes are then exported to the nucleoplasm and, finally, to the cytoplasm. By contrast, self-assembly seems to be an intrinsic property of bacterial ribosomes. ${ }^{2}$

In eukaryotes, with its larger subunits, the additional proteins and RNA expansion segments are located mostly at the periphery of the ribosome $^{3,4}$ (see Figure 2). Their functional roles remain largely uncharacterised, but it is likely that they are involved in the extra degree of translational regulation and control required by all multicellular organisms. Eukaryotic systems present more than 50 extra nucleotide sequences (ie expansion segments) inserted at the conserved rRNA core. It is known that the removal or alteration of some of these segments interferes with the assembly and stability of the rRNA and ribosomal subunits. ${ }^{5,6}$ The eukaryotic ribosomal proteins also participate in the processing of the rRNA during ribosome biogenesis and are known to be essential for cell viability. $^{7}$ In addition, the proteins also present extra-ribosomal functions. A well-characterised example is the highly conserved receptor for activated C-kinase 1 (RACK1), located on the back of the head of the small subunit. ${ }^{8}$ RACK1 functions in a variety of processes, not only participating in the assembly or transport and localisation of the ribosomal particles, but also coupling signal transduction pathways with the synthesis of proteins. ${ }^{9}$

Since the discovery of the ribosome in the mid-1950s, extensive research has been carried out on ribosomal structure and morphogenesis, as well as on the mechanism of its action and regulation. Yet, despite the wide-scale efforts and widespread interest, our knowledge of the mechanisms governing protein synthesis by the ribosome has remained incomplete. Progress toward an understanding of the mechanism of translation in bacteria is of great importance in fighting debilitating pathogenic diseases, as it has the potential to provide clues for the synthesis and application of semi-synthetic (ie derived for natural sources) or totally synthetic antibiotics against drug-resistant pathogenic strains. ${ }^{10}$ Several human disorders linked to disruptions of the protein translation process, including those caused by mutations in specific mRNAs, tRNAs or the ribosome itself, have been described throughout these studies. Among the mutations that affect human ribosomal components is Diamond-Blackfan anaemia, ${ }^{4}$ which is caused by alterations in ribosomal protein S19. Human bone marrow failure syndromes related to mutations of genes that encode additional ribosomal proteins also include Shwachman-Diamond disease, cartilage-hair hypoplasia, dyskeratosis congenita and the Treacher-Collins and $5 q_{-}$-syndromes. ${ }^{11,12}$

During the past decade, several structural studies have described the ribosome's architecture with increasing precision and resolution. X-ray crystallography has been enormously successful as an approach to solve the structure of certain ribosomal complexes, ${ }^{13}$ but this technique runs into limitations when it comes to the analysis of the inherently dynamic behaviour of the ribosome, as many transient structures are very difficult to trap and 


\section{A}
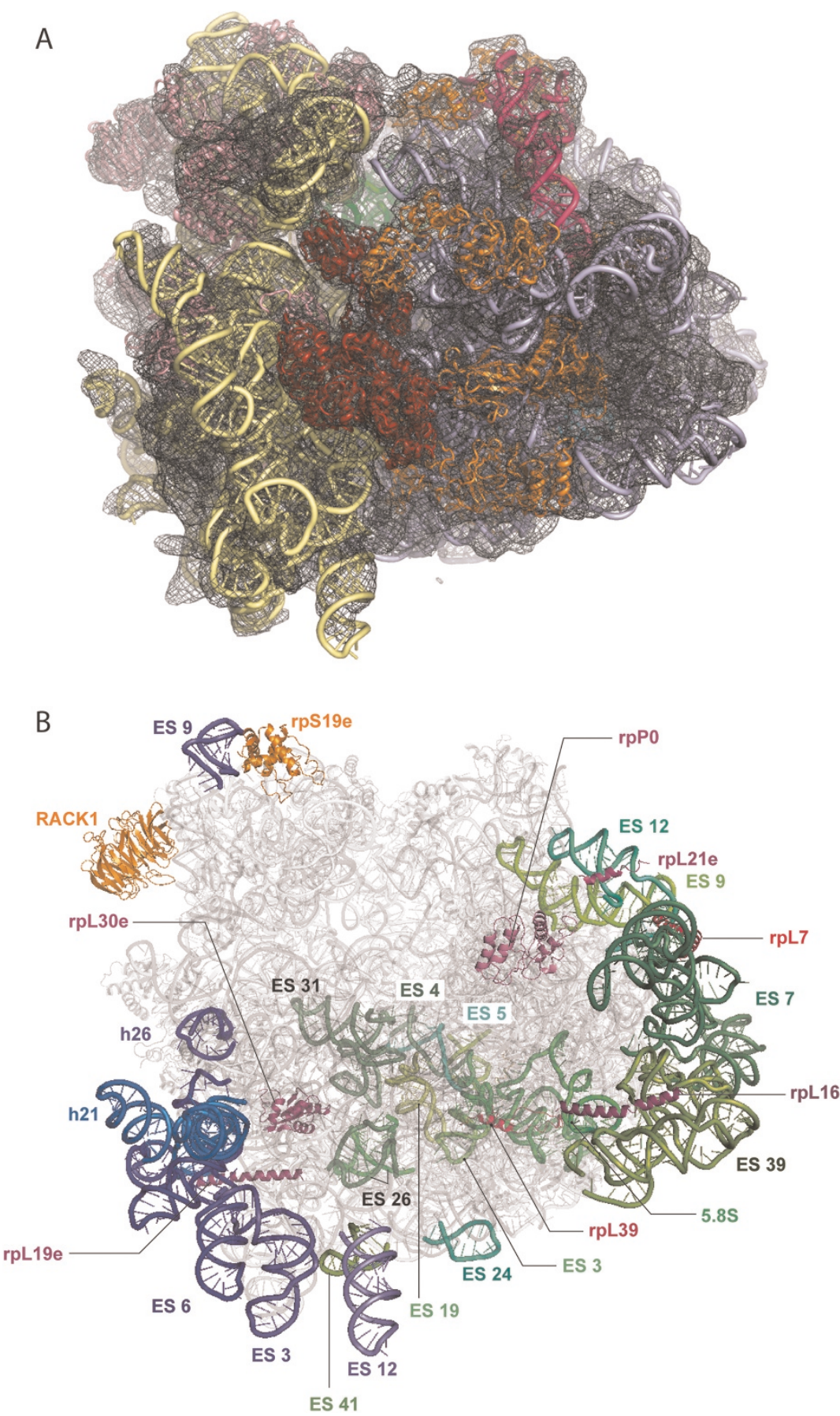

Figure 2. The structure of the eukaryotic ribosome derived from cryo-EM. (A) The atomic model for the yeast $80 \mathrm{~S}$ ribosome was obtained by building on the X-ray structure of the $E$. coli ribosome using rRNA modelling of expansion segments, homology modelling of proteins for which there are bacterial counterparts, and placement of some extra $80 \mathrm{~S}$ proteins whose structure is known. ${ }^{4}$ Experimental cryo-EM densities corresponding to 40S and 60S subunits and eEF2 for Thermomyces lanuginosus, used as constraints for modelling, are shown in transparent yellow, blue and red, respectively. (B) Same as (A) but with extra proteins highlighted (orange, 40S; magenta, 60S), for which the structures are known and which could be located through cross-linking or by exhaustive computational search. (Note that helices marked rpL19e, rpL2/e, rpL7, rpLI6, and rpLI6 have bacterial/archaeal homologues represented in the available X-ray structures for the largest portion of the proteins.) Marked are only pieces (a-helices) supported by EM density proximal to the corresponding protein and by secondary structure predictions.) Likewise, rRNA expansion segments and 5.8S rRNA are highlighted (blue, 40S; green, 60S). In (B), eEF2 has been omitted for clarity. The illustration in (A) was reproduced from Frank (2009). 
crystallise. By contrast, the results coming from cryo-electron microscopy (cryo-EM) data, albeit originally at lower resolution, are highly informative in functional terms when obtained from ribosomes captured in the process of performing their work. When interpreted with the aid of existing $\mathrm{X}$-ray structures, these data provide very accurate three-dimensional (3D) information on each of the elements implicated in the course of the reaction, as well as on the way they are dynamically coupled. Thus cryo-EM studies, in conjunction with other biophysical techniques such as fluorescence stopped-flow and quench-flow analysis or singlemolecule fluorescent resonance energy transfer (sm-FRET), have helped to shape our present understanding of two highly complex dynamic processes during the elongation cycle - decoding and mRNA-tRNA translocation.

\section{Cryo-EM as means to study molecular machines: A short discourse}

In the single-particle method of cryo-EM, the molecule is embedded in a thin $(\sim 1,000 \AA)$ vitreous layer of ice and kept at the temperature of liquid nitrogen for the duration of the experiment (see Figure 3). Cryo-EM is able to image the molecule in a fully hydrated state, without causing deformations. The complex being studied is free to assume all functional states without any steric constraints, allowing, in principle, the visualisation of the entire dynamic course of macromolecular interactions. Time resolution can be achieved by taking 'snapshots' of the complex in successive states of its work cycle, by experimentally 'trapping' these states through chemical or physical means. Because of the low contrast of the molecule in ice and the need to keep the electron dose at very low levels in order to minimise the radiation damage, the resulting projection images are extremely noisy. To overcome the noise, images of multiple 'copies' of the molecule, assumed to have identical structure, must be averaged. The assumption of structural homogeneity is frequently not valid, however, due to the molecule's inherent conformational variability

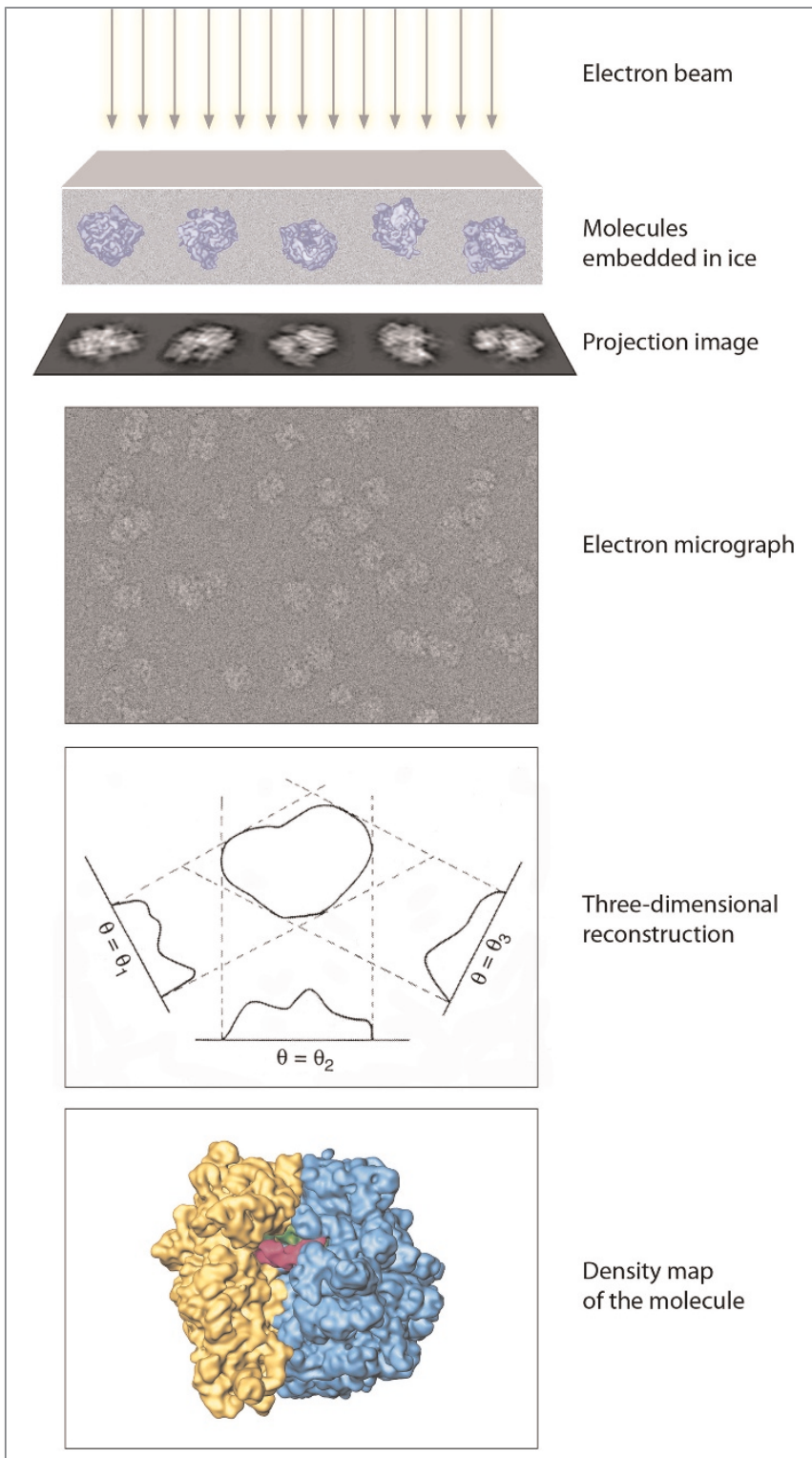

Figure 3. Principle of cryo-EM and single-particle reconstruction. Molecules (in this case ribosomes) lying in random orientations are embedded in a thin layer of ice. Exposure to a low-dose electron beam in the transmission electron microscope produces a projection image ('electron micrograph'). A typical electron micrograph shows $E$. coli ribosomes as low-contrast single particles on a noisy background. After the orientations of the particles have been determined, usually by matching them with a reference, they are used to reconstruct a density map by a back-projection or a similar reconstruction algorithm. This density map is segmented into the different components (subunits, ligands), and the different components are displayed using different colours in a surface representation (bottom panel; small and large subunits are shown in yellow and blue, respectively. A- and P-site tRNAs are coloured pink and green, respectively). 
and non-stoichiometric binding propensity, so classification methodology has to be used to separate the different sub-populations. Once these are identified and separated, individual 3D reconstructions can be generated for each of the individual conformers or binding states.

The current drawback of this approach, which might be gradually overcome with time, is that the spatial resolution of the resulting reconstructions is limited, with the best resolution for asymmetric molecules in the range of 5-7 $\AA .^{14-16}$ Higher resolutions, coming close to the atomic level, have only been achieved for molecules with high symmetry, where symmetry averaging could be applied. ${ }^{17}$ In many cases, however, the atomic structures of most of the players in the molecular binding interactions of a complex are known from $\mathrm{X}$-ray crystallography, or have at least become inferable from related X-ray structures by homology modelling based on sequence comparisons. When all the individual components of a macromolecular complex are known to atomic resolution, the lower-resolution map of the complex will then allow the components to be placed and their atomic interactions to be inferred. Thus, fully to exploit the potential of cryo-EM, it is necessary to fit and dock the X-ray coordinates into the lowerresolution cryo-EM maps. Recent examples for atomic models obtained by such "hybrid" methods are the E. coli ${ }^{18}$ and yeast ribosomes.

\section{Dynamics of the ribosome during protein synthesis: A gallery of changes}

All aspects of translation involve dynamic events. The whole translation apparatus must be viewed as a machinery that is dynamically assembled (during initiation), that engages functional ligands in a cyclic way (during elongation) and that is dynamically disassembled (during termination and recycling). In fact, it is now recognised that the ribosome is a Brownian motor whose main source of energy comes from the thermal environment. ${ }^{19,20}$ sm-FRET has given us a sense of the ribosome as a molecule in constant motion, fluctuating between different states ${ }^{21-23}$ that represent local minima of a complex free-energy landscape. Conformations observable in ensemble averages provided by cryo-EM or X-ray crystallography reflect molecules trapped in those energy minima. The more sharply we can distinguish single-particle projections by classification, the better we will be able to map the entire landscape, toward an understanding of how the ribosome works.

\section{mRNA-tRNA translocation}

The first discovery of a large conformational change of the ribosome during translation was made by cryo-EM. ${ }^{24}$ In the pre-translocational state of the ribosome (ie the state immediately after peptidyl transfer), the A-site tRNA is bound to the polypeptide chain, and the P-site tRNA is deacylated. Upon EF-G binding to the pretranslocational ribosome, the small subunit of the ribosome was found to rotate with respect to the large subunit in a counter-clockwise direction around an axis running normal to the plane separating the subunits (Figure 4A, left). During this so-called ratchet motion, several inter-subunit bridges are remodelled. As originally proposed by Spirin, ${ }^{25}$ this motion has been shown to be essential for EF-G-based mRNA-tRNA translocation. $^{26}$ In recent years, there have been many reports from studies showing a similar conformational change in response to the binding of a number of other factors (IF2, ${ }^{27}$ class-II release factor $3[\mathrm{RF} 3]^{28,29}$ and ribosome recycling factor $[\mathrm{RRF}]{ }^{30}$ see Frank et al. ${ }^{31}$ and Agirrezabala and Frank ${ }^{32}$ ). However, the EF-Tu-bound posttranslocational ribosomes do not display this change, ${ }^{33,34}$ apparently, since the polypeptide bound to the P-site tRNA stabilises the ribosome in the 'normal' conformation. Interestingly, as discussed below, this feature serves to prevent EF-G from binding to a ribosome in the 'wrong', posttranslocational state.

Subsequent research by bulk ${ }^{35}$ and sm-FRET ${ }^{36}$ revealed that the binding of a factor is not required for the pre-translocational ribosome to switch its 
A
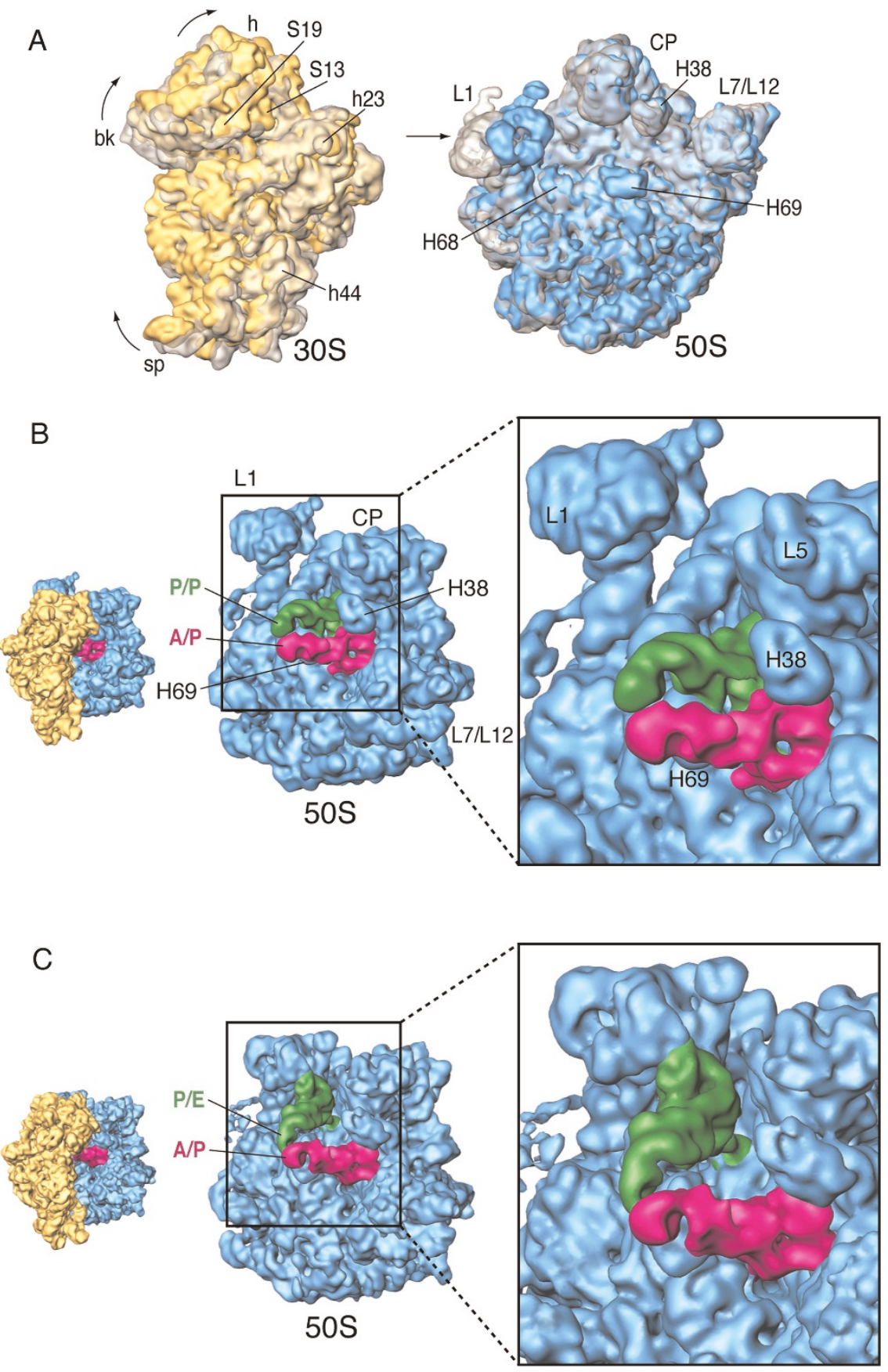

Figure 4. Ratchet-like motion and hybrid tRNA configuration. (A) Superimposition of $30 \mathrm{~S}$ (left) and $50 \mathrm{~S}$ (right) subunits, as seen from the inter-subunit side. Classic-state subunits are shown in transparent grey, while the subunits of the hybrid-state ribosome are in solid yellow (30S) and blue (50S). (B) Close-up view of the 50S subunit showing the classic tRNA configuration. (C) Close-up view of the $50 S$ subunit showing the hybrid tRNA configuration. The orientations of the subunits are shown as successive thumbnails on the left. Data reproduced from Agirrezabala et al. ${ }^{37}$

conformation - under suitable conditions (low $\mathrm{Mg}^{2+}$ concentration), this transition occurs spontaneously, apparently as a result of thermal Brownian motions. Indeed, recently, cryo-EM studies have provided the first structural evidence for the occurrence of spontaneous ratcheting, ${ }^{37,38}$ in agreement with the findings of independent FRET studies. ${ }^{39-42}$ These studies highlight the fact 
that the rearrangement is in fact inherent to the ribosome's labile architecture: the subunits are coupled such that relative rotation requires little expenditure of energy, which is readily supplied by thermal agitation in the surrounding substrate.

Following up with a closer look at the structures, the cryo-EM maps ${ }^{37,38}$ also brought the first visual evidence for an important event of the translocation process (first inferred from footprinting studies by Moazed and Noller), ${ }^{43}$ the formation of the hybrid state of tRNA binding, a state that precedes the complete translocation of the tRNA-mRNA moiety. In this configuration, the acceptor ends of the tRNAs interact with the $\mathrm{P}$ and $\mathrm{E}$ sites of the large subunit, while the anticodon stem loops (ASLs) of the tRNAs still reside in the $\mathrm{A}$ and $\mathrm{P}$ sites of the small subunit, respectively. It is noteworthy that earlier cryo-EM work had already visualised the hybrid $\mathrm{P} / \mathrm{E}$ configuration, but exclusively in EF-G-bound ribosomes with a single deacylated tRNA. ${ }^{44}$ It is now understood, on the basis of the new cryo-EM work, that the new configuration is predisposed by a rearrangement of proteins S13, L1 and L5 and helices 68, 69 and 38 of 23S rRNA elements that alter their relative location concurrently with the spontaneous ratchet motion (Figure 4B,C). In a newly completed study, X-ray crystallography of pre-translocational complexes has produced structures of ribosomes in an intermediate state of ratcheting. ${ }^{45}$ These structural intermediates, albeit obtained using ASL tRNA mimics instead of complete tRNA molecules, are shedding light on the way that the tRNAs acquire the hybrid configuration in atomic detail.

In the cryo-EM studies, the ratcheting of the small subunit is observed to be coupled with the movement of another very dynamic component of the translation machinery, the L1 stalk. This structural element of the large subunit is seen in two different positions, open and closed relative to the body of the large subunit (Figure 4A right). Like a gate-keeper, the L1 stalk is located at the end of the tRNA's pathway. In its closed position (which coincides with the ratcheted position of the small subunit ${ }^{44}$ ), the L1 stalk is displaced toward the inter-subunit space, in essence blocking the exit as it makes contact with the $\mathrm{P} / \mathrm{E}$ hybrid tRNA. Recent sm-FRET experiments have identified three positions of the L1 stalk. ${ }^{46}$ These conformations, open, half-closed and closed, are coupled to the tRNA configuration. New studies suggest the existence of an allosteric collaboration between the L1 stalk and EF-G during tRNA translocation and, possibly, during the release of the E-site tRNA from the ribosome. ${ }^{47}$

Other relevant illustrative examples of dynamic features essential for tRNA translocation are the bending movement of helix 44 (see Figure 4A, left), causing a displacement of its vertex (where the decoding centre is located) toward the $\mathrm{P}$ site by $\sim 8 \AA,{ }^{48}$ as well as the small subunit's head rotation (or 'swivelling') with respect to the body. The rotation, which proceeds toward the E site, parallels the trajectory of the tRNAs through the ribosome. This rearrangement accounts for the remaining distance of $10-12 \AA$ that separates the $\mathrm{A}$ and $\mathrm{P}$ sites after the afore-mentioned bending of helix 44 . Observed first in cryo-EM reconstructions of eEF2-bound $80 \mathrm{~S}$ ribosomes, ${ }^{49,50}$ the relevance of such a movement for the completion of mRNAtRNA translocation has been reiterated based on the observation of different head orientations in crystal structures of vacant ribosomes. ${ }^{51}$

Of fundamental importance in all translational activity is the recruitment of translational factors by the ribosome. Some of these factors perform their catalysis in a GTP-dependent manner (IF2, EF-Tu and EF-G, and RF3 in eubacteria). Several lines of research show that the recruitment and GTP-hydrolysis activity of all these factors is facilitated by the so-called L7/L12 stalk (see Figure 4A, right), a very flexible structural component located at the side opposite of the L1 stalk. ${ }^{52,53}$ This stalk, formed by protein L10 and multiple copies of L7/ 12 (with the exact number depending on the organism), protrudes laterally from the ribosome and changes its conformation in the course of elongation. Due to its mobility and highly dynamic nature, the electron density corresponding to the stalk is not generally seen in the structures of either isolated 50S subunits or complete $70 \mathrm{~S}$ ribosomal structures; however, cryo-EM studies, combined 
with atomic coordinates of its individual components, have helped to delineate its structure and suggested a mechanism of action. ${ }^{54}$

\section{Decoding}

Of particular interest are the dynamic events of decoding, crucial for the high-fidelity synthesis of proteins. The structural basis of mRNA decoding

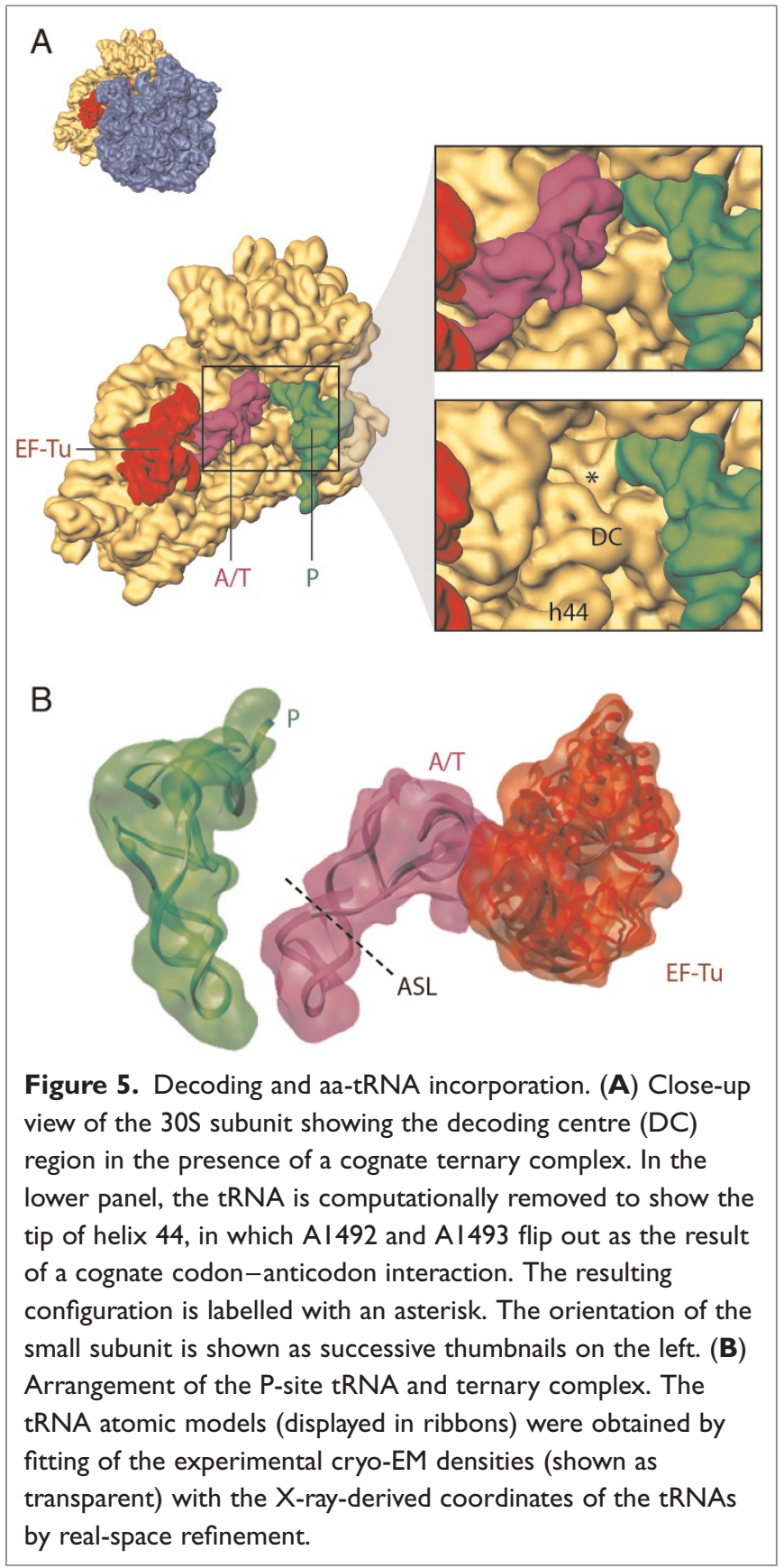

by the ribosome was elucidated by X-ray crystallography of $30 \mathrm{~S}$ subunits bound with a tRNA fragment in the presence of an antibiotic. ${ }^{55}$ These data show that nucleotides G530, and A1492 and A1493 from helix 44 of $16 \mathrm{~S}$ rRNA monitor the correctness of the Watson-Crick geometry of the helix formed by the base pairing of codon and anticodon. When the correct (ie corresponding to cognate pairing) geometry is recognised, these bases form firm contacts with the minor groove of the helix. An ensuing global change of head and shoulder domains, referred to as domain closure, is the most prominent signature of a cognate match. ${ }^{56}$

Cryo-EM has greatly contributed toward expansion of our knowledge about the delivery of the incoming aa-tRNA to the A site, as well as of the mechanism of GTPase activation that follows the cognate recognition. ${ }^{33,34,57,58}$ The analysis by cryo-EM of ternary aa-tRNA-EFTu-GTP complexes stalled on the ribosome (using specific antibiotics) in the so-called $\mathrm{A} / \mathrm{T}$ state revealed that the aa-tRNA molecule presents a distorted conformation as it enters the ribosome (see Figure 5). The bending distortion is localised at positions 44, 45 and 26 of the tRNA, and is associated with a $\sim 45$ degree twist of the ASL. This configuration is pivotal for the cognate aa-tRNA selection, as it allows Watson-Crick pairing of the codon with the anticodon. In addition, the GTPase-associated centre, placed at the base of the L7/L12 stalk and formed by protein L11 and a fragment of helices 43 and 44 of $23 \mathrm{~S}$ rRNA, is seen to be displaced toward the body of the large subunit. As this motion goes hand in hand with the distortion of the tRNA, this element, located on the large subunit, appears to have a role during decoding, a process which is otherwise mainly orchestrated by the small subunit.

One of the most fascinating problems in translation is the nature of the conformational signal reporting on the successful cognate match at the decoding centre and travelling to the active region of EF-Tu. It is still an open question if the signal is transmitted through the tRNA itself, through a contact between the $30 \mathrm{~S}$ subunit and EF-Tu, or by a combination of these pathways (see Li et al., ${ }^{34}$ 
Ogle and Ramakrishnan, ${ }^{59}$, Cochella and Green ${ }^{60,}$ and Schmeing et al. ${ }^{61}$ for further insights).

\section{Peptide bond formation}

For completeness, the structural basis of peptide bond formation should briefly be mentioned. Little is known from cryo-EM data due to resolution limitations; however, X-ray crystallography studies have characterised the structure of the peptidyltransferase centre in great detail, highlighting the structural basis for its catalytic mechanism. According to the present view, the rapid ribosomeinduced peptidyl-transfer reaction $\left(\sim 10^{7}\right.$-fold enhancement ${ }^{62}$ ) is facilitated by local conformational changes in the cavity where the peptidyl moiety is positioned upon binding of the A-site substrate. $^{63,64}$ This model invokes an induced-fit mechanism involving nucleotides 2553, 2585 and 2506 (Escherichia coli numbering), in charge of aligning the reactive substrates - that is, the $\alpha$-amino group of the incoming amino acid and the terminal carboxyl group of the P-site tRNA (for more details, see Simonovic and Steitz ${ }^{65}$ and references therein).

\section{Conclusions and future perspectives}

The field of ribosome research has undergone astonishing progress in recent years, due to our increasingly acute insights into the structural basis of the translational machinery. Advances in both $\mathrm{X}$-ray crystallography and cryo-EM of the ribosome have provided extensive information on the structure and dynamics of ribosomal subunits and functional ligands, their changing binding constellations and mechanism of action. A revolution of sorts has taken place in the understanding of ribosomal interaction with its ligands, paralleling the change in the understanding of enzyme kinetics elsewhere. It is now recognised that in the thermal environment, the structures of both host and ligand molecules are in constant flux, and that the final conformation of the host in which we find the ligand bound already pre-exists within the whole range of its dynamically changing conformations.
Most strikingly, as discussed above, both cryo-EM and sm-FRET show evidence that, after peptidyl transfer, the ribosome constantly fluctuates between two major conformations, and that one of these is stabilised by the binding of EF-G, an event that triggers GTP hydrolysis and renders translocation irreversible. Other examples are the spontaneous fluctuations of the L1 stalk between (at least) three functional states, probably coupled to the binding and movement of tRNAs along the inter-subunit cavity, or the changes in the decoding centre and the ternary complex triggered upon cognate codon-anticodon match.

Compared with what is known for bacteria, the eukaryotic ribosome is still a largely uncharted territory. Due to the absence of crystals suitable for X-ray crystallography, despite many efforts of several laboratories, the only structural information has come from density maps from cryo-EM. ${ }^{3,4,49,50,66-69}$ On this basis, some atomic models have been built that depict the interesting evolutionary development of peripheral, nonconserved regions. ${ }^{3,4,70}$ As far as the mechanism of translation during the elongation cycle is concerned, there is evidence for yeast that mRNAtRNA translocation is facilitated by the same large-scale structural reorganisation and EF-binding mechanism as in bacteria. ${ }^{49,50,71}$ Considering that the most ambitious goal is the understanding of regulation and control of the human ribosome, particularly the various dysfunctions of these mechanisms in human disease, a very long and tedious path of discovery is still ahead.

\section{Acknowledgments}

We thank Lila Iino-Rubenstein for assistance with the preparation of the illustrations. This work was supported by HHMI and NIH grants R01 GM55440 and R37 GM29169 (to J.F.). X.A is a recipient of a 'Ramon y Cajal' fellowship from the Spanish Government.

\section{References}

1. Acker, M.G and Lorsch, J.R. (2008), 'Mechanism of ribosomal joining during eukaryotic translation initiation', Biochem. Soc. Trans. Vol. 36, pp. 653-657.

2. Dinman, J.D. (2008), 'The eukaryotic ribosome: Current status and challenges', J. Biol. Chem. Vol. 284, pp. 11761-11765. 
3. Spahn, C.M., Beckmann, R., Eswar, N. et al. (2001), 'Structure of the $80 \mathrm{~S}$ ribosome from Saccharomyces cerevisiae - tRNA-ribosome and subunit-subunit interactions', Cell Vol. 107, pp. 373-386.

4. Taylor, D.J., Devkota, B., Huang, A.D. et al. (2009), 'Comprehensive molecular structure of the eukaryotic ribosome', Structure Vol. 17, pp. $1591-1604$

5. Musters, W., Boon, C.A., van der Sande, H. et al. (1990), 'Functional analysis of transcribed spacers of yeast ribosomal DNA', EMBO J. Vol. 9 , pp. 3989-3996.

6. Sweeney, R.L., Chen, L. and Yao, M.C. (1994), 'An rRNA variable region has an evolutionarily conserved essential role despite sequence divergence', Mol. Cell Biol. Vol. 14, pp. 4203-4215.

7. Ferreira-Cerca, S.G., Poll, G., Gleizes, P.E. et al. (2005), 'Roles of eukaryotic ribosomal proteins in maturation and transport of pre-18S rRNA and ribosome function', Mol. Cell Vol. 20, pp. 263-275.

8. Sengupta, J., Nilsson, J., Gursky, R. et al. (2004), 'Identification of the versatile scaffold protein RACK1 on the eukaryotic ribosome by cryo-EM', Nat. Struct. Mol. Biol. Vol. 11, pp. 957-962.

9. Nilsson, J., Sengupta, J., Frank, J. et al. (2004), 'Regulation of eukaryotic translation by the RACK1 protein: a platform for signalling molecules on the ribosome', EMBO Rep. Vol. 5, pp. 1137-1141.

10. Tenson, T. and Mankin, A. (2006), 'Antibiotics and the ribosome', Mol. Microbiol. Vol. 59, pp. 1664-1677.

11. Scheper, G.C., van der Knaap, M.S. and Proud, C.G. (2007), 'Translation matters: Protein synthesis defects in inherited disease', Nat. Rev. Genet. Vol. 8, pp. 711-723.

12. Narla, A. and Ebert, B.L. (2010), 'Ribosomopathies: Human disorders of ribosome dysfunction', Blood, in press.

13. Schmeing, T.M. and Ramakrishnan, V. (2009), 'What recent ribosome structures have revealed about the mechanism of translation', Nature Vol. 461, pp. 1234-1242.

14. LeBarron, J., Grassucci, R.A., Shaikh, T.R. et al. (2008), 'Exploration of parameters in cryo-EM leading to an improved density map of the E. coli ribosome', J. Struct. Biol. Vol. 164, pp. 24-32.

15. Schuette, J.C., Murphy, FV. IV, Kelley, A.C. et al. (2009), 'GTPase activation of elongation factor EF-Tu by the ribosome during decoding', EMBO J. Vol. 28, pp. 755-765.

16. Seidelt, B., Innis, C.A., Wilson, D.N. et al. (2009), 'Structural insight into nascent polypeptide chain-mediated translational stalling', Science Vol. 326, pp. 1412-1415.

17. Yu, X., Yin, L. and Zhou, Z.H. (2008), '3.88§ structure of cytoplasmic polyhedrosis virus by cryo-electron microscopy', Nature Vol. 453, pp. 415-419.

18. Trabuco, L.G., Villa, E., Mitra, K. et al. (2008), 'Flexible fitting of atomic structures into electron microscopy maps using molecular dynamics', Structure Vol. 16, pp. 673-683.

19. Spirin, A.S. (2009), 'The ribosome as a conveying thermal ratchet machine', J. Biol. Chem. Vol. 284, pp. 21103-21119.

20. Frank, J. and Gonzalez, R.L. (2010), 'Structure and dynamics of a processive Brownian motor: The translating ribosome', Ann. Rev. Biochem., in press.

21. Marshall, R.A., Aitken, C.E., Dorywalska, M. et al. (2008a), 'Translation at the single-molecule level', Annu. Rev. Biochem. Vol. 77, pp. 177-203.

22. Munro, J.B., Vaiana, A., Sanbonmatsu, K.Y. et al. (2008), 'A new view of protein synthesis: Mapping the free energy landscape of the ribosome using single-molecule FRET', Biopolymers Vol. 89, pp. 565-577.

23. Munro, J.B., Sanbonmatsu, K.Y., Spahn, C.M. et al. (2009), 'Navigating the ribosome's metastable energy landscape', Trends. Biochem. Sci. Vol. 34, pp. 390-400.

24. Frank, J. and Agrawal, R.G. (2000), 'A ratchet-like inter-subunit reorganization of the ribosome during translocation', Nature Vol. 406, pp. 318-322.

25. Spirin, A.S. (1968), 'How does the ribosome work? A hypothesis based on the two subunit construction of the ribosome', Curr. Mod. Biol. Vol. 2, pp. $115-127$

26. Horan, L.H. and Noller, H.F. (2007), 'Intersubunit movement is required for ribosomal translocation', Proc. Natl. Acad. Sci. USA Vol. 104, pp. $4881-4885$
27. Allen, G.S., Zavialov, A., Gursky, R. et al. (2005), 'The cryo-EM structure of a translation initiation complex from Escherichia coli', Cell Vol. 121, pp. 703-712.

28. Klaholz, B.P., Myasnikov, A.G. and Van Heel, M. (2004), 'Visualization of release factor 3 on the ribosome during termination of protein synthesis', Nature Vol. 427, pp. 862-865.

29. Gao, H., Zhou, Z., Rawat, U. et al. (2007), 'RF3 induces ribosomal conformational changes responsible for dissociation of class I release factors', Cell Vol. 129, pp. 929-941.

30. Gao, N., Zavialov, A.V., Li, W. et al. (2005), 'Mechanism for the disassembly of the posttermination complex inferred from cryo-EM studies', Mol. Cell Vol. 18, pp. 663-674.

31. Frank, J., Gao, H., Sengupta, J. et al. (2007), 'The process of mRNA-tRNA translocation', Proc. Natl. Acad. Sci. USA Vol. 104, pp. 19671-19678.

32. Agirrezabala, X. and Frank, J. (2009), 'Elongation in translation as a dynamic interaction between the ribosome, tRNA, and elongation factors EF-G and EF-Tu', Q. Rev. Biophys. Vol. 3, pp. 159-200.

33. Valle, M., Zavialov, A., Li, W. et al. (2003), 'Incorporation of aminoacyl-tRNA into the ribosome as seen by cryo-electron microscopy', Nat. Struct. Biol. Vol. 10, pp. 899-906.

34. Li, W., Agirrezabala, X., Lei, J. et al. (2008), 'Recognition of aminoacyl-tRNA: A common molecular mechanism revealed by cryo-EM', EMBO J. Vol. 27, pp. 3322-3331.

35. Ermolenko, D.N., Majumdar, Z.K., Hickerson, R.P. et al. (2007), 'Observation of intersubunit movement of the ribosome in solution using FRET', J. Mol. Biol. Vol. 370, pp. 530-540.

36. Kim, H.D., Puglisi, J.D. and Chu, S. (2007), 'Fluctuations of transfer RNAs between classical and hybrid states', Biophys. J. Vol. 93, pp. 3575-3582.

37. Agirrezabala, X., Lei, J., Brunelle, J.L. et al. (2008), 'Visualization of the hybrid state of tRNA binding promoted by spontaneous ratcheting of the ribosome', Mol. Cell Vol. 32, pp. 190-197.

38. Julian, P., Konevega, S.H., Scheres, S.H. et al. (2008), 'Structure of ratcheted ribosomes with tRNAs in hybrid states', Proc. Natl. Acad. Sci. USA Vol. 105, pp. 16924-16927.

39. Munro, J.B., Altman, R.B., O'Connor, N. et al. (2007), 'Identification of two distinct hybrid state intermediates on the ribosome', Mol. Cell Vol. 25 , pp. $505-517$.

40. Fei, J., Kosuri, P., MacDougall, D.D. et al. (2008), 'Coupling of ribosomal L1 stalk and tRNA dynamics during translation elongation', Mol. Cell Vol. 30, pp. 348-359.

41. Cornish, P.V., Ermolenko, D.N., Noller, H.F. et al. (2008), 'Spontaneous intersubunit rotation in single ribosomes', Mol. Cell Vol. 30, pp. $578-588$.

42. Marshall, R.A., Dorywalska, M. and Puglisi, J.D. (2008), 'Irreversible chemical steps control intersubunit dynamics during translation', Proc. Natl. Acad. Sci. USA Vol. 105, pp. 15364-15369.

43. Moazed, D. and Noller, H.F (1989), 'Intermediate states in the movement of transfer RNA in the ribosome', Nature Vol. 342, pp. 142-148.

44. Valle, M., Zavialov, A., Sengupta, J. et al. (2003), 'Locking and unlocking of ribosomal motions', Cell Vol. 114, pp. 123-134.

45. Zhang, W., Dunkle, J.A. and Cate, J.H. (2009), 'Structures of the ribosome in intermediate states of ratcheting', Science Vol. 325, pp. 1014-1017.

46. Cornish, P.V., Ermolenko, D.N., Staple, D.W. et al. (2009), 'Following movement of the L1 stalk between three functional states in single ribosomes', Proc. Natl. Acad. Sci. USA Vol. 106, pp. 2571-2576.

47. Sternberg, S.H., Fei, J., Prywes, N. et al. (2009), 'Translation factors direct intrinsic ribosome dynamics during translation termination and ribosome recycling', Nat. Struct. Mol. Biol. Vol. 16, pp. 861-868.

48. Van Loock, M.S., Agrawal, R.K., Gabashvili, I.S. et al. (2000), 'Movement of the decoding region of the $16 \mathrm{~S}$ ribosomal RNA accompanies tRNA translocation', J. Mol. Biol. Vol. 304, pp. 507-515.

49. Spahn, C.M., Gomez-Lorenzo, M.G., Grassucci, R.A. et al. (2004), 'Domain movements of elongation factor eEF2 and the eukaryotic 80S ribosome facilitate tRNA translocation', EMBO J. Vol. 23, pp. $1008-1019$. 
50. Taylor, D.J., Nilsson, J., Merrill, A.R. et al. (2007), 'Structures of modified eEF2 80S ribosome complexes reveal the role of GTP hydrolysis in translocation', EMBO J. Vol. 26, pp. 2421-2431.

51. Schuwirth, B.S, Borovinskaya, M.A., Hau, C.W. et al. (2005), 'Structures of the bacterial ribosome at 3.5 A resolution', Science Vol. 310, pp. $827-834$.

52. Mohr, D.W., Wintermeyer, W. and Rodnina, M.V. (2002), 'GTPase activation of elongation factors $\mathrm{Tu}$ and $\mathrm{G}$ on the ribosome', Biochemistry Vol. 41, pp. $12520-12528$.

53. Helgstrand, M., Mandava, C.S., Mulder, F.A. et al. (2007), 'The ribosomal stalk binds to translation factors IF2, EF-Tu, EF-G and RF3 via a conserved region of the L12 C-terminal domain', J. Mol. Biol. Vol. 365, pp. $468-479$.

54. Diaconu, M., Kothe, U., Schlunzen, F. et al. (2005), 'Structural basis for the function of the ribosomal L7/12 stalk in factor binding and GTPase activation', Cell Vol. 121, pp. 991-1004.

55. Ogle, J.M., Brodersen, D.E., Clemons, W.M. et al. (2001), 'Recognition of cognate transfer RNA by the 30 S ribosomal subunit', Science Vol. 292, pp. 897-902.

56. Ogle, J.M., Murphy, FV. IV, Tarry, M.J. et al. (2002), 'Selection of tRNA by the ribosome requires a transition from an open to a closed form', Cell Vol. 111, pp. 721-732.

57. Villa, E., Sengupta, J., Trabuco, L.G. et al. (2009), 'Ribosome-induced changes in elongation factor Tu conformation control GTP hydrolysis', Proc. Natl. Acad. Sci. USA Vol. 106, pp. 1063-1068.

58. Schuette, J.C., Murphy, F.V. IV, Kelley, A.C. et al. (2009), 'GTPase activation of elongation factor EF-Tu by the ribosome during decoding', EMBO J. Vol. 28, pp. 755-765

59. Ogle, J.M. and Ramakrishnan, V. (2005), 'Structural insights into translational fidelity', Annu. Rev. Biochem. Vol. 74, pp. 129-177.

60. Cochella, L. and Green, R. (2005), 'An active role for tRNA in decoding beyond codon:anticodon pairing', Science Vol. 308, pp. 1178-1180.
61. Schmeing, T.M., Voorhees, R.M., Kelley, A.C. et al. (2009), 'The crystal structure of the ribosome bound to EF-Tu and aminoacyl-tRNA', Science Vol. 326, pp. 688-694.

62. Beringer, M. and Rodnina, M. (2007), 'The ribosome peptidyl transferase', Mol. Cell Vol. 26, pp. 311-321.

63. Schmeing, T.M., Huang, K.S., Strobel, S.A. et al. (2005), 'An induced-fit mechanism to promote peptide bond formation and exclude hydrolysis of peptidyl-tRNA', Nature Vol. 438, pp. 520-524.

64. Vorhees, R.M., Weixlbaumer, A., Loakes, D. et al. (2009), 'Insights into substrate stabilization from snapshots of the peptidyl transferase center of the intact 70 S ribosome', Nat. Struct. Mol. Biol. Vol. 16, pp. 528-533.

65. Simonovic, M. and Steitz, T.A. (2009), 'A structural view on the mechanism of the ribosome-catalyzed peptide bond formation', Biochim. Biophys. Acta Vol. 1789, pp. 612-623.

66. Beckmann, R., Spahn, C.M.., Eswar, N. et al. (2001), 'Architecture of the protein-conducting channel associated with the translating $80 \mathrm{~S}$ ribosome', Cell Vol. 107, pp. 361-372.

67. Morgan, D.G., Menetret, J.F., Neuhof, A. et al. (2002), 'Structure of the mammalian ribosome-channel complex at 17A resolution', J. Mol. Biol. Vol. 324, pp. 871-886.

68. Spahn, C.M., Jan, E., Mulder, A. et al. (2004), 'Cryo-EM visualization of a viral internal ribosome entry site bound to human ribosomes: The IRES functions as an RNA-based translation factor', Cell Vol. 118, pp. 465-475.

69. Halic, M., Gartmann, M., Schlenker, O. et al. (2006), 'Signal recognition particle receptor exposes the ribosomal translocon binding site', Science Vol. 312, pp. 745-747.

70. Chandramouli, P.M., Topf, M., Menetret, J.F. et al. (2008), 'Structure of the mammalian $80 \mathrm{~S}$ ribosome at 8.7 A resolution', Structure Vol. 16, pp. $535-548$.

71. Sengupta, J., Nilsson, J., Gursky, R. et al. (2008), 'Visualization of the eEF2-80S ribosome transition-state complex by cryo-electron microscopy', J. Mol. Biol. Vol. 382, pp. 179-187. 